\title{
ON THE EXISTENCE OF CROSS SECTIONS IN LOCALLY FLAT BUNDLES
}

GUIDO KARRER

1. A principal bundle $\mathfrak{B}(X, G)$ with base space $X$ and group $G$ is called "locally flat" if the structural group $G$ can be reduced to a totally disconnected subgroup $G_{1} \subset G$, or in other words: if there exists a totally disconnected subgroup $G_{1} \subset G$, a principal bundle $\mathfrak{B}_{1}\left(X, G_{1}\right)$ and an injection $j: \mathfrak{B}_{1} \rightarrow \mathfrak{B}[1]$. For instance, if a differentiable bundle $\mathfrak{B}$ admits an infinitesimal connection whose curvature form is identically zero, then $\mathfrak{B}$ is locally flat $[1$, Reduction theorem, p. 37].

We are mainly interested in locally flat bundles with arcwise connected structural group $G$. The aim of this note is to give necessary and sufficient conditions for locally flat bundles to have a cross section.

2. We have to make use of certain more or less obvious properties of locally flat bundles which were discussed in a previous paper. As all bundles in the sequel will be principal bundles we shall omit the word "principal"; we assume all base spaces $X$ of bundles to be arcwise connected.

Definition. A reduction of a bundle $\mathfrak{B}(X, G)$ is the collection of a subgroup $G_{1} \subset G$, bundle $\mathfrak{B}_{1}\left(X, G_{1}\right)$ and injection $j: \mathfrak{B}_{1} \rightarrow \mathfrak{B}$. Suppose $G$ can be reduced to a totally disconnected subgroup $G_{1} \subset G$. Corresponding to points of reference $x_{0} \in X$ and $b_{1} \in B_{1}$, where $B_{1}$ is the bundle space of $\mathfrak{B}_{1}$, there exists the characteristic homomorphism $\chi: \pi_{1}(X) \rightarrow G_{1}[2$, p. 61$]$. We say that the reduction is "irreducible" if $\chi$ is onto.

Definition. Let $j: \mathfrak{B}_{1}\left(X, G_{1}\right) \rightarrow \mathfrak{B}(X, G)$ be a reduction, $G_{1}$ totally disconnected. $\chi: \pi_{1}(X) \rightarrow G_{1}$ is called a "characteristic homomorphism of the reduction," $H=\operatorname{ker}(\chi)$ the "kernel of the reduction."

3. From now on we assume $X$ to be arcwise connected, arcwise locally connected and semi locally 1 -connected. This will make sure the existence of bundles over $X$ with totally disconnected group and prescribed characteristic homomorphism. In particular, to every invariant subgroup $N \subset \pi_{1}(X)$ belongs a regular covering $p_{2}: \tilde{X} \rightarrow X$ such that $\pi_{1}(\tilde{X}) \approx N$. If we put $P=\pi_{1}(X) / N$ then $\tilde{X}$ is the space of a bundle $\mathfrak{B}_{2}(X, P)$ with discrete group $P$, and the right action of $p \in P$ on $\tilde{x} \in \tilde{X}$-denoted by $\tilde{x} \cdot p$-is a "deckbewegung."

Received by the editors July $28,1960$. 
TheOREM 1. Let $\mathfrak{B}(X, G)$ be locally flat, $\chi: \pi_{1}(X) \rightarrow G_{1} \subset G$ a characteristic homomorphism and $H$ the kernel of a reduction $j: \mathfrak{B}_{1}\left(X, G_{1}\right)$ $\rightarrow \mathfrak{B}(X, G)$. Let $N$ be an invariant subgroup of $\pi_{1}(X)$ such that $N \subset H$. $\chi$ induces a homomorphism $P \rightarrow G_{1}$ again denoted by $\chi$. Let $\tilde{X}$ be the covering space of $X$ corresponding to $N$. Then, $\mathfrak{B}(X, G)$ has a cross section if and only if there exists a map $f: \tilde{X} \rightarrow G$ such that

$$
f(\tilde{x} \cdot p)=f(\tilde{x}) \chi(p) \quad \text { for all } \tilde{x} \in \tilde{X} \text { and } p \in P .
$$

THEOREM 2. Suppose a homomorphism $\chi: \pi_{1}(X) \rightarrow G_{1} \subset G$ is given, and $G_{1}$ is totally disconnected. Then there exist bundles $\mathfrak{B}(X, G)$, $\mathfrak{B}_{1}\left(X, G_{1}\right)$ and injection $j: \mathfrak{B}_{1} \rightarrow \mathfrak{B}$ such that $\chi$ is a characteristic homomorphism of this reduction. Furthermore, any two such bundles $\mathfrak{B}(X, G)$ are equivalent.

For the proof of Theorems 1 and 2 we refer to [3].

4. Definition. Let $T^{n}$ denote the $n$-dimensional torus, $n \geqq 0 ; T^{0}$ consists of one point. Let $G$ be a topological group and $G_{1}$ a subgroup $\subset G$. We say that $G_{1}$ is $n$-flat in $G$ if every bundle $\mathfrak{B}\left(T^{n}, G\right)$ over $T^{n}$ whose structural group $G$ can be reduced to $G_{1}$, has a cross section.

Clearly, $G_{1}=\{e\}$ is $n$-flat in $G$ for every $n$. If $G$ is arcwise connected, every subgroup of $G$ is 1 -flat in $G$. If $G_{1}$ is $n$-flat in $G$, then $G_{1}$ is $p$-flat in $G$ for every $0 \leqq p \leqq n$. As will be shown later, $n$-flat subgroups which are totally disconnected are characterized by topological properties.

Theorem 3. Suppose $X$ is, in addition to being connected in the sense of $\S 3$, a normal space with the property that every covering of $X$ by open sets is reducible to a countable covering. Let $\mathfrak{B}(X, G)$ be locally flat, $j: \mathfrak{B}_{1}\left(X, G_{1}\right) \rightarrow \mathfrak{B}(X, G)$ a reduction, and $H$ the kernel of the reduction. Suppose there exists an invariant subgroup $N \subset \pi_{1}(X)$ such that $N \subset H$ and $P=\pi_{1}(X) / N$ is a finitely generated free abelian group whose dimension is $n$. Then $\mathfrak{B}$ has a cross section if $G_{1}$ is $n$-flat in $G$.

5. The proof of Theorem 3 will be preceded by two lemmas.

Lemma 1. Let $X$ and $N \subset \pi_{1}(X)$ be the same as in Theorem 3. Let $p_{2}: \tilde{X} \rightarrow X$ be the covering corresponding to $N,\left\{p_{1}, \cdots, p_{n}\right\}$ a basis of $P=\pi_{1}(X) / N,\left\{e_{1}, \cdots, e_{n}\right\}$ a basis of Euclidean space $E^{n}$. The correspondence $p_{i} \rightarrow e_{i}$ sets up an isomorphism $\psi: P \rightarrow Z^{n}$, where $Z^{n}$ is the vector group generated by $\left\{e_{1}, \cdots, e_{n}\right\}$. Then, there exists a map $t: \tilde{X} \rightarrow E^{n}$ such that $t(\tilde{x} \cdot p)=t(\tilde{x})+\psi(p)$.

The composition of the natural homomorphism $\phi: \pi_{1}(X) \rightarrow P$ and $\psi$ is a homomorphism $\psi_{\phi}: \pi_{1}(X) \rightarrow Z^{n} \subset E_{a}^{n}, E_{a}^{n}$ being the additive group 
of $E^{n}$. By Theorem 2 there exist bundles $\mathfrak{B}\left(X, E_{a}^{n}\right), \mathfrak{B}_{1}\left(X, Z^{n}\right)$ and injection $j: \mathfrak{B}_{1} \rightarrow \mathfrak{B}$, such that $\psi_{\phi}$ is a characteristic homomorphism of this reduction. The topological space of $E_{a}^{n}$ is solid and $X$ is such that every bundle over $X$ with a solid fibre has a cross section [2, p. 55]. Hence, $\mathfrak{B}\left(X, E_{a}^{n}\right)$ has a cross section and the map $t$ of the lemma is the map $f$ given by Theorem 1, the right hand side of (3.1) written additively and $\chi$ replaced by $\psi$.

Lemma 2. The situation being as in Theorem 3 and Lemma 1, let $T^{n}$ be the $n$-dimensional torus and $\left\{u_{1}, \cdots, u_{n}\right\}$ a basis of $\pi_{1}\left(T^{n}\right) . E^{n}$ is the universal covering space of $T^{n}$, and we can assume that the lifting of curves in the class $u_{i}$ provides curves with initial point $O \in E^{n}$ and endpoint $e_{i} \in E^{n}$. This induces an isomorphism $\pi_{1}\left(T^{n}\right) \approx Z^{n}$, with respect to which $\chi_{1}=\chi \psi^{-1}$ can also be interpreted as a homomorphism $\pi_{1}\left(T^{n}\right) \rightarrow G_{1}$. By Theorem 2 there exist bundles $\mathfrak{B}\left(T^{n}, G\right), \mathfrak{B}_{1}\left(T^{n}, G_{1}\right)$ and injection $j_{1}: \mathfrak{B}_{1} \rightarrow \mathfrak{B}$ such that $\chi_{1}$ is a characteristic homomorphism of this reduction.

Suppose now, $G_{1}$ is $n$-flat in $G$. Then the bundle $\mathscr{B}\left(T^{n}, G\right)$ has a cross section which, on the other hand, means that there exists a map $f_{1}: E^{n} \rightarrow G$ such that $f_{1}(v+z)=f_{1}(v) \chi_{1}(z)$ for all $v \in E^{n}$ and $z \in Z^{n}$. The composition of this $f_{1}$ with $t$ given by Lemma 1 is a map $f=f_{1} t: \tilde{X} \rightarrow G$ which has the property $(3.1)$. Hence $\mathfrak{B}(X, G)$ has a cross section and Theorem 3 is proved.

6. Theorem 4. Let the notations be as before. Assume the reduction in Theorem 3 to be irreducible. Suppose there exists a map $s: E^{n} \rightarrow X$ such that $s(v+z)=s(v) \cdot \psi^{-1}(z)$ for all $v \in E^{n}$ and $z \in Z^{n}$. Then $\mathfrak{B}(X, G)$ has a cross section if and only if $G_{1}$ is $n$-flat in $G$.

The sufficiency is given by Theorem 3 . Let $\mathfrak{B}(X, G)$ have a cross section. There exists a map $f: \tilde{X} \rightarrow G$ with the property (3.1). The composition of $f$ with $s$ is a map $f_{1}=f s: E^{n} \rightarrow G$ such that $f_{1}(v+z)$ $=f_{1}(v) \chi_{1}(z)$ for all $v \in E^{n}$ and $z \in Z^{n}$. This means that $\mathfrak{B}\left(T^{n}, G\right)$ has a cross section (Lemma 2 and Theorem 1). The proof of Theorem 4 will be complete after the correctness of the following has been shown.

Lemma 3. A totally disconnected subgroup $G_{1} \subset G$ is $n$-flat in $G$ if there exist bundles $\mathfrak{B}\left(T^{n}, G\right), \mathfrak{B}_{1}\left(T^{n}, G_{1}\right)$ and an irreducible reduction $j_{1}: \mathfrak{B}_{1} \rightarrow \mathfrak{B}$ (we mean by this that all characteristic homomorphisms $\chi_{1}: \pi_{1}\left(T^{n}\right) \rightarrow G$ are onto) such that $\mathfrak{B}\left(T^{n}, G\right)$ has a cross section.

The lemma will be proved later. We are going to explain now the notion of $n$-flatness in detail.

7. In the following, $G_{1} \subset G$ will always be assumed to be totally 
disconnected. Let $\left\{h_{1}, \cdots, h_{n}\right\}$ be a commutative set of $n$ elements in $G_{1}$. We mean by this a set of $n$ elements $h_{i} \in G_{1}$ such that $h_{i} h_{k}$ $=h_{k} h_{i}$. Let $\left\{e_{1}, \cdots, e_{n}\right\}$ be a basis of Euclidean space $E^{n}$.

Lemma 4. If $G_{1}$ is $n$-flat in $G(n \geqq 1)$, then there exists a map $f: E^{n} \rightarrow G$ such that

$$
f\left(v+e_{i}\right)=f(v) h_{i} \quad \text { for all } v \in E^{n} \text { and } i=1, \cdots, n .
$$

If $\left\{p_{1}, \cdots, p_{n}\right\}$ is a basis of $\pi_{1}\left(T^{n}\right)$ we can assume that the lifting of curves in the class $p_{i}$ provides curves from $0 \in E^{n}$ to $e_{i} \in E^{n}$. The correspondence $p_{i} \rightarrow h_{i}$ generates a homomorphism $\chi_{1}: \pi_{1}\left(T^{n}\right) \rightarrow G_{1}$. By Theorem 2 there exists a bundle $\mathfrak{B}\left(T^{n}, G\right)$ and a reduction of $G$ to $G_{1}$ with characteristic homomorphism $\chi_{1}$. If $G_{1}$ is $n$-flat in $G, \mathfrak{B}\left(T^{n}, G\right)$ has a cross section, and $f$ in Lemma 4 is the $\operatorname{map} f$ given by Theorem 1 .

Let now $T^{n}(n \geqq 0)$ be the $n$-dimensional torus and $G$ a topological group. Let $t_{0} \in T^{n}$ be a point of reference and $F^{n}$ the set of continuous mappings $f:\left(T^{n}, t_{0}\right) \rightarrow(G, e)$. If $f_{1} \in F^{n}, f_{2} \in F^{n}$, then $f_{1} \cdot f_{2}$ denotes the mapping $f_{1} \cdot f_{2}(t)=f_{1}(t) \cdot f_{2}(t), t \in T^{n}$, and $f_{1}^{-1}$ the composition of $f_{1}: T^{n} \rightarrow G$ with the map $g \rightarrow g^{-1}$ in $G$. Let $\tau_{n}(G)$ denote the set of homotopy classes of mappings $f \in F . \tau_{n}(G)$ is a group, the product of two classes $u_{1}$ and $u_{2}$ being defined as the class of products $f_{1} \cdot f_{2}$, $f_{1} \in u_{1}, f_{2} \in u_{2}$. Clearly, $\tau_{1}(G)=\pi_{1}(G)=$ fundamental group of $G$.

Suppose then $\left\{h_{1}, \cdots, h_{n}\right\}$ is a commutative set of elements of $G_{1}$. If $G_{1}$ is $n$-flat in $G$ and $\left\{e_{1}, \cdots, e_{n}\right\}$ is a basis of $E^{n}$ there exists a map $f: E^{n} \rightarrow G$ with property (7.1). We may even assume

$$
f(0)=e=\text { identity in } G,
$$

because, if $f$ satisfies (7.1) and $a=f(0)$ then $g(v)=a^{-1} \cdot f(v)$ satisfies both (7.1) and (7.2). This being said, let $h_{n+1} \in G_{1}$ be such that $h_{i} h_{n+1}=h_{n+1} \cdot h_{i}, i=1, \cdots, n$. Because of the commutativity, $h_{n+1}^{-1} \cdot f$ $\cdot h_{n+1}: E^{n} \rightarrow G$ will again have properties (7.1) and (7.2). If two mappings $f_{1}: E^{n} \rightarrow G$ and $f_{2}: E^{n} \rightarrow G$ have properties (7.1) and (7.2), then $f_{1} \cdot f_{2}^{-1}: E^{n} \rightarrow G$ induces a map $\bar{g}:\left(T^{n}, t_{0}\right) \rightarrow(G, e)$ where $t_{0}=p_{2}(0)$ and $p_{2}: E^{n} \rightarrow T^{n}$ is the covering, such that $f_{1} \cdot f_{2}^{-1}=\bar{g} p_{2}$. Hence $h_{n+1}^{-1} \cdot f \cdot h_{n+1}$ $\cdot f^{-1}$ induces a map $\bar{f}:\left(T^{n}, t_{0}\right) \rightarrow(G, e)$ which lies in a homotopy class $u \in \tau_{n}(G)$. If the elements $h_{1}, \cdots, h_{n+1}$ are kept fixed, $u$ still might depend on $f$. Let $f_{1}: E^{n} \rightarrow G$ be another map with properties (7.1) and (7.2), and $\bar{f}_{1}$ be the map $\in F^{n}$ induced by $h_{n+1}^{-1} \cdot f_{1} \cdot h_{n+1} \cdot f_{1}^{-1}$. Applying the reasoning above, $f_{1} \cdot f^{-1}=g$ induces a map $\bar{g} \in F^{n}$. From $h_{n+1}^{-1} \cdot f_{1}$ $\cdot h_{n+1} \cdot f_{1}^{-1}=h_{n+1}^{-1} \cdot g \cdot h_{n+1} \cdot h_{n+1}^{-1} \cdot f \cdot h_{n+1} \cdot f^{-1} \cdot g$ follows $\bar{f}_{1}=h_{n+1}^{-1} \cdot \bar{g} \cdot h_{n+1} \cdot \bar{f}$ $\cdot \bar{g}^{-1}$. As $h_{n+1}^{-1} \cdot \bar{g} \cdot h_{n+1}$ is homotopic to $\bar{g}$ in $F^{n}$ one gets $u_{1}=u_{g} \cdot u \cdot u_{0}^{-1}$, where $u_{1}, u_{0}$ is the homotopy class of $\bar{f}_{1}, \bar{g}$ resp. In short, we have the following result. 
Lемма 5. Let $G_{1}$ be $n$-flat in $G, n \geqq 1$. The construction above assigns to every commutative set of $(n+1)$ elements $h_{i} \in G_{1}, i=1, \cdots,(n+1)$, an equivalence class $\psi_{n}\left(h_{1}, \cdots, h_{n+1}\right)$ under inner automorphisms of elements $\in \tau_{n}(G)$.

8. We adopt the convention that, if $\psi$ denotes an equivalence class under inner automorphisms of elements of a group $K, \psi=0$ means it contains the identity $e \in K$.

TheOREM 5. A totally disconnected group $G_{1} \subset G$ is $(n+1)$-flat in $G(n \geqq 1)$, if and only if it is $n$-flat in $G$ and $\psi_{n}\left(h_{1}, \cdots, h_{n+1}\right)=0$ for every commutative set of elements in $G_{1}$. The statement: $G_{1}$ is 1-flat in $G$, is equivalent to: $G_{1}$ lies in the arcwise connected component of $e \in G$.

The second part of Theorem 5 is obvious. As for the first part, let us assume that $G_{1}$ is $n$-flat in $G(n \geqq 1)$ and $\psi_{n}\left(h_{1}, \cdots, h_{n+1}\right)=0$. Let $\mathfrak{B}\left(T^{n+1}, G\right), \mathfrak{B}_{1}\left(T^{n+1}, G_{1}\right)$ be bundles, $j: \mathfrak{B}_{1} \rightarrow \mathfrak{B}$ an injection and $\chi: \pi_{1}\left(T^{n+1}\right) \rightarrow G_{1}$ a characteristic homomorphism of this reduction. Let $p_{2}: E^{n+1} \rightarrow T^{n+1}$ be the universal covering, $\left\{p_{1}, \cdots, p_{n+1}\right\}$ a basis of $\pi_{1}\left(T^{n+1}\right),\left\{e_{1}, \cdots, e_{n+1}\right\}$ a basis of $E^{n+1}$ such that the lifting of closed curves in the class $p_{i} \in \pi_{1}\left(T^{n+1}, t_{0}\right)$ provides curves in $E^{n+1}$ from 0 to $e_{i}, t_{0}=p_{2}(0)$. The set of elements $h_{i}=\chi\left(p_{i}\right) \in G_{1}, i=1, \cdots,(n+1)$, is certainly commutative. As $G_{1}$ is $n$-flat in $G$ there exists by Lemma 4 a map $f_{1}: E^{n} \rightarrow G$ such that

$$
f_{1}\left(v+e_{i}\right)=f_{1}(v) h_{i}, \quad \text { for all } v \in E^{n} \text { and } i=1, \cdots, n,
$$

holds. Here $E^{n}$ denotes the space spanned by the vectors $e_{1}, \cdots, e_{n}$. We may assume $f_{1}(0)=e \in G$ (7.2). Denote by $g: E^{n} \rightarrow G$ the map $h_{n+1}^{-1} \cdot f_{1} \cdot h_{n+1} \cdot f_{1}^{-1}$, by $\bar{g}: T^{n} \rightarrow G$ the induced map such that $g=\bar{g} p_{2}^{1}$, where $p_{2}^{1}: E^{n} \rightarrow T^{n}$ is the covering map. By the assumption $\psi_{n}\left(h_{1}, \cdots, h_{n+1}\right)=0$ there exists a homotopy $\bar{h}:\left(I \times T^{n}, t_{0}\right) \rightarrow(G, e)$ such that $\bar{h}(1, t)=\bar{g}(t)$ and $\bar{h}(0, t)=e$ for $t \in T^{n}$. Then $h$ defined by $h(\rho, v)=\bar{h}\left(\rho, p_{2}^{1}(v)\right)$ for $\rho \in I$ and $v \in E^{n}$, is a homotopy of $g$ such that

$$
\begin{aligned}
h(1, v)=g(v), h(0, v)=e=h(\rho, 0), h\left(\rho, v+e_{i}\right) & =h(\rho, v), \\
i & =1, \cdots, n .
\end{aligned}
$$

Let now $w: I \rightarrow G$ be a curve connecting $e=w(0)$ and $w(1)=h_{n+1}$. Then $f^{*}$ defined by $f^{*}(\rho, v)=w(\rho) h(\rho, v) f_{1}(v)$ is a mapping $I \times E^{n} \rightarrow G$ which has the following properties

$$
\begin{aligned}
& f^{*}(0, v)=f_{1}(v), \quad f^{*}\left(\rho, v+e_{i}\right)=f^{*}(\rho, v) h_{i} \quad(i=1, \cdots, n), \\
& f^{*}(1, v)=f^{*}(0, v) h_{n+1} .
\end{aligned}
$$

Any vector $w \in E^{n+1}$ can be written as $\rho e_{n+1}+v, v \in E^{n}$. Let $m$ be an 
integer and $E_{m}$ the set $\left\{w=\rho e_{n+1}+v \mid m \leqq \rho \leqq(m+1)\right\}$. We define mappings $f_{m}: E_{m} \rightarrow G$ by $f_{m}(w)=f^{*}(\rho-m, v) h_{n+1}^{m}$. Because of (8.2) one has $f_{m}(w)=f_{m+1}(w)$ if $w \in E_{m} \cap E_{m+1}$, thus the collection $\left\{f_{m}\right\}$ determines one map $f: E^{n+1} \rightarrow G$ with the property $f\left(w+e_{i}\right)=f(w) h_{i}, i=1, \cdots$, $(n+1)$, where $h_{i}=\chi\left(p_{i}\right)=\chi\left(e_{i}\right)$. But the existence of such a map asserts the existence of a cross section in $\mathfrak{B}\left(T^{n+1}, G\right)$ (Theorem 1 ). Hence $G_{1}$ is $(n+1)$-flat in $G$.

Conversely suppose $G_{1}$ to be $(n+1)$-flat in $G(n \geqq 1)$. As was pointed out in connection with the definition of $n$-flatness, $(n+1)$-flatness induces $n$-flatness. Let $\left\{h_{1}, \cdots, h_{n+1}\right\}$ be a commutative set of elements in $G_{1}$. Using the same notations as before, the correspondence $p_{i} \in \pi_{1}\left(T^{n+1}\right) \rightarrow h_{i}$ generates a homomorphism $\chi: \pi_{1}\left(T^{n+1}\right) \rightarrow G_{1}$ into. By Theorem 2, $\chi$ is a characteristic homomorphism of a certain reduction $j: \mathfrak{B}_{1}\left(T^{n+1}, G_{1}\right) \rightarrow \mathfrak{B}\left(T^{n+1}, G\right)$ where $\mathfrak{B}\left(T^{n+1}, G\right)$ has a cross section because of the assumed $(n+1)$-flatness of $G_{1}$ in $G$. By Theorem 1 there exists a map $f: E^{n+1} \rightarrow G$ such that $f\left(w+e_{i}\right)=f(w) h_{i}$ for $w \in E^{n+1}$ and $i=1, \cdots,(n+1)$. Denote by $f_{1}$ the restriction $f \mid E^{n}$. We may assume $f_{1}(0)=e(7.2)$. As $w=\rho e_{n+1}+v, v \in E^{n}$, we can write $f(w)=f_{\rho}(v)$. Denote by $u(\rho)$ the expression $f_{\rho}(0)=f\left(\rho e_{n+1}\right)$. Then $h$ defined by $h(\rho, v)=u^{-1}(\rho) f_{\rho}(v) f_{1}^{-1}(v)$ is continuous in $E^{n+1}$ and represents for $0 \leqq \rho \leqq 1$ a homotopy of $h(1, v)=h_{n+1}^{-1} f_{1}(v) h_{n+1} f_{1}^{-1}(v)$ in $h(0, v)$ $=e$. As for all $\rho u^{-1}(\rho) f_{\rho}\left(v+e_{i}\right) f_{1}^{-1}\left(v+e_{i}\right)=u^{-1}(\rho) f_{\rho}(v) f_{1}^{-1}(v), h$ will induce a homotopy $\bar{h}:\left(I \times T^{n}, t_{0}\right) \rightarrow(G, e)$ of the map $\bar{g}:\left(T^{n}, t_{0}\right) \rightarrow(G, e)$ induced by $h_{n+1}^{-1} \cdot f_{1} \cdot h_{n+1} \cdot f^{-1}$ in the map $T^{n} \rightarrow e$. Therefore $\psi_{n}\left(h_{1}, \cdots, h_{n+1}\right)=0$, and the proof of Theorem 5 is complete.

9. There still remains to prove Lemma 3 . The situation is the following: two bundles $\mathfrak{B}\left(T^{n}, G\right), \mathfrak{B}_{1}\left(T^{n}, G_{1}\right)$ together with a reduction $j: \mathfrak{B}_{1} \rightarrow \mathfrak{B}$ are given such that $G_{1} \subset G$ is totally disconnected, $\mathfrak{B}\left(T^{n}, G\right)$ has a cross section and, if $\chi$ denotes a characteristic homomorphism, $\chi: \pi_{1}\left(T^{n}\right) \rightarrow G_{1}$ is onto. Using the same notations as before, there exists a map $f: E^{n} \rightarrow G$ such that $f(v+z)=f(v) \chi(z)$ for $v \in E^{n}$ and $z \in Z^{n}$ (Theorem 1) and $f(0)=e(7.2) . Z^{n}$ is the vector group generated by $\left\{e_{1}, \cdots, e_{n}\right\}$ and as such isomorphic to $\pi_{1}\left(T^{n}\right)$. Any $g_{1} \in G_{1}$ is the image under $\chi$ of a certain $z \in Z^{n}$. Let $g_{1}=\chi(z)$, then $u(\rho)=f(\rho z)$ is a curve in $G$ from $e$ to $g_{1}$. This shows that $G_{1} \subset$ arcwise connected component of $G$ and hence is 1-flat in $G$ (Theorem 5). Suppose $G_{1}$ is already proved to be $p$-flat in $G, 1 \leqq p<n$. Let $\left\{h_{1}, \cdots, h_{p+1}\right\}$ be an ordered set of elements in $G_{1}$; note that $G_{1}$ is abelian. Let $v_{i} \in Z^{n}$ be such that $\chi\left(v_{i}\right)=h_{i}$. If $\left\{e_{1}, \cdots, e_{p+1}\right\}$ is a basis of $E^{p+1}$ then the correspondence $e_{i} \rightarrow v_{i}$ generates a linear map $\phi$ of $E^{p+1}$ onto the vectorspace spanned by $\left\{v_{1}, \cdots, v_{p+1}\right\}$. The composition $f \phi: E^{p+1} \rightarrow G$ has 
the property $f \phi\left(w+e_{i}\right)=f \phi(w) h_{i}$ for $w \in E^{p+1}$ and $i=1, \cdots,(p+1)$. But now we have the same situation as in the second part of the proof of Theorem 5. The reasoning applied there leads to the conclusion $\psi_{p}\left(h_{1}, \cdots, h_{p+1}\right)=0$. It follows from Theorem 5 that $G_{1}$ is $(p+1)$ flat in $G$.

10. CoRollary 1. Every totally disconnected subgroup $G_{1}$ of an arcwise connected abelian group $G$ is $n$-flat in $G$ for every $n$.

This follows directly from Theorem 5. One just has to observe that elements of $\psi_{n}\left(h_{1}, \cdots, h_{n+1}\right)$ are homotopy classes of mappings of the form $h_{n+1}^{-1} \cdot f \cdot h_{n+1} \cdot f^{-1}$.

Corollary 2. Let $X$ be a topological space as in Theorem 3. If $\pi_{1}(X)$ is a finitely generated free abelian group, then every locally flat bundle with base space $X$ and arcwise connected abelian group $G$ has a cross section.

This is a consequence of Theorem 3 and Corollary 1.

11. We wish to give an example of a locally flat bundle without cross section. Let $R^{3}$ denote the group of rotations in Euclidean space $E^{3}$ and $\left\{e_{1}, e_{2}, e_{3}\right\}$ be an orthogonal basis in $E^{3}$. Define $h_{1}$ and $h_{2} \in R^{3}$ by $h_{1}\left(e_{i}\right)=-e_{i}, i=1,2, h_{1}\left(e_{3}\right)=e_{3}, h_{2}\left(e_{1}\right)=e_{1}, h_{2}\left(e_{k}\right)=-e_{k}$ for $k=2,3$. Clearly $h_{1} h_{2}=h_{2} h_{1}$. The set of rotations in $R^{3}$ leaving $e_{3}$ fixed may be identified with $R^{2}$. If $g \in R^{2}$, then $h_{2}^{-1} g h_{2}=g^{-1}$, especially if $f: I \rightarrow R^{2}$ is a curve from $e$ to $h_{1}, h_{2}^{-1} f h_{2}=f^{-1}$. As $h_{1}^{2}=e, u=h_{2}^{-1} \cdot f h_{2} f^{-1}=f^{-2}$ is a closed curve in $R^{2}$, and we can choose $f$ such that the homotopy class of $u$ in $R^{2}$ generates $\pi_{1}\left(R^{2}\right)$. But then $u$ cannot be homotopic to zero in $R^{3}$ either, which means $\psi_{1}\left(h_{1}, h_{2}\right) \neq 0$. Hence the discrete group $G_{1}=\left\{e, h_{1}, h_{2}, h_{1} h_{2}\right\}$ is not 2-flat in $R^{3}$. If $T^{2}$ is now the two-dimensional torus we define $\chi: \pi_{1}\left(T^{2}\right) \rightarrow G_{1}$ by $\chi\left(p_{i}\right)=h_{i}, i=1,2$, where $\left\{p_{1}, p_{2}\right\}$ in a basis of $\pi_{1}\left(T^{2}\right)$. Note that $\chi$ is onto. By Theorem 2 there exist bundles $\mathfrak{B}\left(T^{2}, R^{3}\right), \mathfrak{B}_{1}\left(T^{2}, G_{1}\right)$ and injection $j: \mathfrak{B}_{1} \rightarrow \mathfrak{B}$ such that $\chi$ is a characteristic homomorphism of this reduction. Theorem 4 then asserts that $\mathfrak{B}\left(T^{2}, R^{3}\right)$ does not have a cross section.

\section{REFERENCES}

1. K. Nomizu, Lie groups and differential geometry, Tokyo, The Mathematical Society of Japan, 1956.

2. N. Steenrod, The topology of fibre bundles, Princeton University Press, 1951.

3. G. Karrer, Some properties of locally flat bundles, Michigan Math. J., to appear.

University of Bonn, West Germany 